\title{
Pyruvate Metabolism in Friedreich's Ataxia
}

\author{
A. BARBEAU, R. F. BUTTERWORTH. T. NGO. G. BRETON. S. MELANÇON.
} D. SHAPCOTT, G. GEOFFROY, AND B. LEMIEUX

SUMMARY: Friedreich's ataxia patients show evidence of an abnormally elevated and prolonged response of pyruvate and lactate to a glacose load, with normal fasting levels. However, there is a bimodal distribution of this response with high and low pyruvate responders. This trait appears to be determined genetically, However, although in vivo tests suggest low oxidation of pyruvate, we were unable to confirm any in vitro impairment of each of the components of the pyruvate dehydrogenase $(P D H)$ complex. We conclude that the defect is in the metabolic regulation of $P D H$, probably at the E3 (lipoamide dehydrogenase) step.

RÉSUMÉ: Les patients avec ataxie de Friedreich, en tant que groupe, montrent de façon undubitable une réponse anormalement élevée et prolongée da pyravate et du lactate à une surcharge en glucose, tout en maintenant des taux de base normaux à jeîn. Cependant, il semble y avoir une distribation bimodale de cette réponse, incluant des réponses élevées ou basses. Ce trait semble être déterminé génétiquement. Cependant, alors que les tests in vivo montrent une oxidation lente du pyruvate, par contre les tests in vitro ne semblent pas confirmer une atteinte d'une quelconque des composantes du complexe pyruvate dehydrogenase $(P D H)$. Nous concluons que le défaut est au niveau de la régulation métabolique du PDH, probablement au niveau de la composante $E_{3}$ (Lipoamide dehydrogenase).

From the Clinical Research Institute of Montreal; the Hopital Ste-Justine de Montreal; The Centre Hospitalier Universitaire de Sherbrooke; and the Hôpital Hôtel-Dieu de Montréal.

Reprint requests for the complete supplement on Friedreich's ataxia to: Dr. André Barbeau, Clinical Research Institute of Montreal, 110 Pine Avenue West, Montreal H2W 1R7, Quebec, Canada.

\section{INTRODUCTION}

Previous studies (Thoren, 1962; Hewer and Robinson, 1968; Podolsky and Sheremata, 1970) and our own work (See previous papers) have demonstrated that there is a high incidence of "clinical" and "chemical" diabetes or glucose intolerance in many types of ataxias, including Friedreich's, with or without changes in insulin secretion or reactivity to a glucose load. This fact was known before the start of the present study and therefore a number of metabolic evaluations of the glucose metabolic pathway in Friedreich's ataxia were included in the common protocol. Among these was an evaluation of pyruvate and lactate metabolism. This was based on previously reported findings of abnormal pyruvate handling in motor neuron disease (Shahani et al., 1971), a variety of neuromuscular disorders (Collis and Engel, 1968), obesity, polyneuritis (Joiner et al., 1950) and in many patients with intermittent ataxia (Blass et al., 1971). During the early phase of our evaluations, Kark et al. (1974) published a paper demonstrating deficient pyruvate oxidation in neuromuscular diseases, including Friedreich's ataxia. This was the impetus for a more detailed investigation of this aspect in some of the patients included in our survey.

Pyruvate oxidation is an extremely complex biochemical phenomenon which has been shown by Reed and his co-workers (Reed and Cox, 1966; Reed, 1974) to comprise a successive cascade of at least three enzymatic steps under the regulatory control of two more enzymes. At each of these steps cofactors also play an important role. The first component of the pyruvate dehydrogenase (PDH) complex is pyruvate decarboxylase $\left(E_{1}\right)$, a thiamine pyrophosphosphate (TPP) dependent enzyme. The second step $\left(E_{2}\right)$ is the enzyme lipoyl reductase transacetylase. The last step $\left(E_{3}\right)$ is dehydrolipoyl dehydrogenase also called lipoamide dehydrogenase (LAD) employing flavin-adeninedinucleotide (FAD) as co-factor and under the control of the nicotinamide-adenine-dinucleotide (NAD)-(NADH) reaction (Figure 1 ). The function of LAD is to regenerate oxidized lipoic acid. Finally, this tri-enzyme complex can be found in the active or inactive states, transformation between the two states being dependent upon a kinase (to phosphorylated, inactive state) or a phosphatase (to dephosphorylated, active state) and the divalent ions calcium and magnesium (Figure 2). In the presence of calcium, the phosphatase binds to the transacetylase moiety favoring dephosphorylation. The present paper will take a preliminary look at each of these steps in a large sample of patients with typical Friedreich's ataxia.

\section{SUBJECTS AND METHODS}

The patients studied were mostly from Group la (typical Friedreich's ataxia), but also included examples from other sub-groups and were chosen according to availability at the time each individual test was planned. Appropriate age-matched controls were also chosen for each test.

a) Glucose-pyruvate test: Following the ingestion of two consecutive doses (30' apart) of $50 \%$ glucose (50 $\mathrm{ml}$ in $200 \mathrm{ml} \mathrm{H}_{2} \mathrm{O}$ ), blood was withdrawn at $0,60,90$ and $120 \mathrm{~min}$. Pyruvate and lactate were measured by U.V. spectrophotometer at 340 $\mathrm{m} \mu$ according to standard proce- 
dures (Joiner et al., 1950). The patients were tested in the morning, fasting, after 3 days of high carbohydrate intake. Calculations and comparisons were made at time 0 and every subsequent time of blood drawing (Student's t-test). Areas under the curve were also measured: total area by planimetry, "abnormal" area by planimetry of the surface under the curve but over a stated baseline of $100 \mu \mathrm{M} / \mathrm{l}$, and finally the difference between the results at $120^{\prime}$ and $0^{\prime}(\Delta 120-0)$. Similar plots and calculations were made for lactate.

b) Thiamine pyrophosphate (T.P.P.), the co-factor requirement of the first step of the pyruvate de- hydrogenase complex (PDH); pyruvate decarboxylase $\left(E_{1}\right)$, was measured using the transketolase method of Dreyfus (P. Dreyfus, personal communication). This is intended to evaluate deficiencies in thiamine (Vit. B-1) by using D-xylulose-5-PO as source of active glycoaldehyde and measuring the formation of sedoheptulose-5- $\mathrm{PO}_{4}$ in a HitachiPerkin-Elmer 139 spectrophotometer. This determination is done with and without the addition of excess TPP (2 mM).

c) The pyruvate dehydrogenase components were measured, in white blood cells (leukocytes) and cultured fibroblasts, by incubation of $1-{ }^{14} \mathrm{C}, 2-{ }^{14} \mathrm{C}$ or uniformly label-

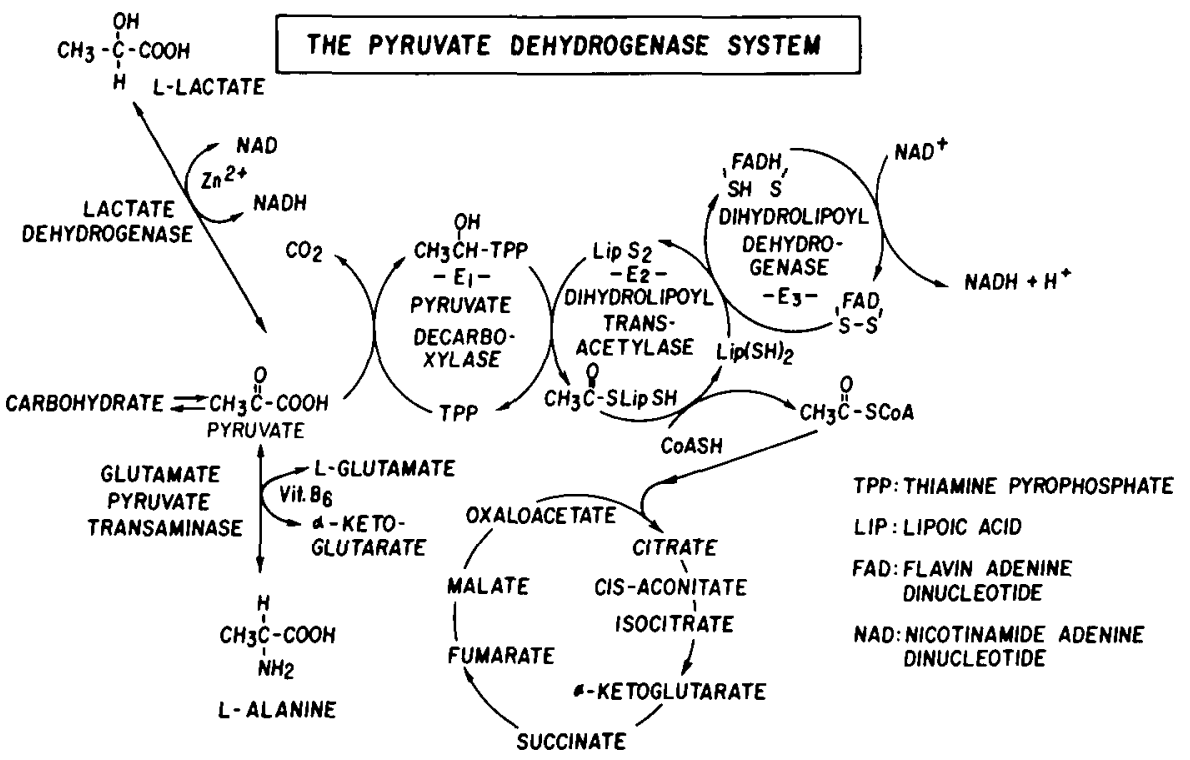

Figure 1 - The pyruvate dehydrogenase (PDH) complex.

REGULATION OF THE PYRUVATE DEHYDROGENASE COMPLEX

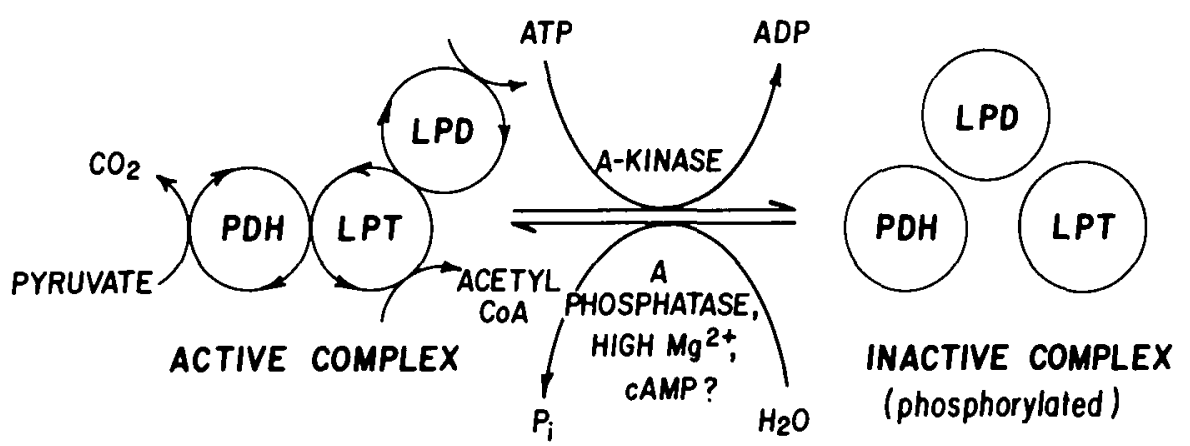

Figure 2 - Regulation of the pyruvate dehydrogenase complex. led ${ }^{14} \mathrm{C}$-pyruvic acid (New England Nuclear Corporation) and capture of the liberated ${ }^{14} \mathrm{CO}_{2}$ with standard methods similar to those employed by Blass and collaborators (1976). These results gave measures of pyruvate oxidation (see Discussion). Fibroblasts were cultured according to the method of Strömme et al. (1976).

d) The final component, dehydrolipoyl-dehydrogenase (lipoamide dehydrogenase, LAD) was measured in serum using modifications of the method recently described by Pelley et al. (1976).

e) Serum alanine determinations were carried out by chromatography (as described in the previous paper). f) The serum enzymes glutamic pyruvate transaminase (SGPT) and lactic dehydrogenase (LDH) were measured by routine laboratory procedures including the automated SMA $15 / 60$.

\section{RESULTS}

a) Pyruvate-glucose test

Initially, we carried out this test indiscriminately on 21 consecutive ataxic patients (from any groups la, $\mathrm{Ila}$, and IIb), and in 16 normal agematched control subjects from our laboratories. It can be seen in Figure 3 that the ataxic subjects are significantly different from the control group in their pyruvate response to glucose. This is true at all times after the ingestion, despite the fact that initial fasting levels were not different between the 2 groups. The same type of response can be observed for blood lactate levels (Figure 4), but in this case fasting levels in the ataxics are higher. These results have been reported (Barbeau, 1975).

A reevaluation of these findings, with the addition of 2 cases was then carried out. The new sample comprised 17 Group la patients with typical Friedreich's ataxia, 6 Group IIa patients (atypical Friedreich possibly recessive Roussy-Levy syndrome), 5 subjects with the dominant form of spino-cerebellar degeneration, and 6 neurological controls (2 motor neuron disease, 1 Parkinson's disease and 3 myopathies). The results are com- 
piled in Table 1. It is seen that the neurological controls and the dominant spino-cerebellar degenerations are not significantly different from normal controls as far as their pyruvate response to glucose is concerned (measured as abnormal surface under the curve over and above the upper limit, or threshold, of normal values). The response of the 17 Group Ia patients is abnormal, exagerated and delayed in its return to normal. This is also true, but to a lesser extent in Group Ila patients. The response of lactate to glucose in all these patients followed essentially the same pattern, but is much more affected by a large number of variables, therefore is less reliable.

Perusal of the individual results gave the impression that the Group Ia subjects did not form a heterogeneous sample, but included at least 2 different sub-groups (Figure 5) which we could call the low and the high pyruvate response subgroups. If this division is genetically determined, we should be able to delineate familial clusters of such responses. It was possible to study 5 kindreds from group la cases (Table 2), including 4 asymptomatic siblings of the 10 ataxic patients, and their 10 asymptomatic parents. The apparently normal siblings did not differ from the normal control subjects. The 10 parents, as a group, had higher values. In fact, the means of the 3 groups (normals, parents, ataxics) are almost exactly in a 1:3:6 ratio $\left(758 ; 2321 ; 5200 \mathrm{~mm}^{2}\right)$, with the parents half-way to the values observed in their ataxic children. This pattern is compatible with the autosomal recessive transmission of a biochemical defect reflected in abnormal responses of pyruvate to a glucose load. Within this small sample, a clear-cut delineation of two sub-groups could be made. Four patients had distinctly elevated pyruvate responses to glucose $\left(5000 \mathrm{~mm}^{2}\right.$ and over) while 6 patients had low responses $\left(0-5000 \mathrm{~mm}^{2}\right)$. The highresponders were from 3 kindreds, the low responders from 2 other kindreds. There were no overlaping values. We looked at the responses of their parents. Again the division into high and low responders was clear and always consistent with the pattern observed in their ataxic children. The low responder parents, however, were not within normal limits, their pyruvate response being significatively lower than in normal controls. In all these findings, the responses of lactate were in parallel.

The biochemical characteristics of the two groups of responses are compared in Table 3 and will be discussed later. Only $4 / 6$ patients in the low group are included, because a brother and sister of one case were studied for pyruvate response, but were not part of the total protocol. Some trends are evident, even in this small sample. The high pyruvate responders have a higher $2 \mathrm{hr}$. P.C. glucose, a higher Danowski score (oral GTT analysis), and a lower fasting insulin level. Their verbal I.Q. is consistently lower. Both groups have higher than normal mean total bilirubin levels, this being more evident in the low responders.

TABLE 1

PYRUVATE-GLUCOSE TEST IN ATAXIAS

(mean \pm S.D.)

\begin{tabular}{|c|c|c|c|}
\hline & & ABNORMAL PYRUVATE & ABNORMAL LACTATE \\
\hline GROUP & $\underline{N}$ & $\frac{\text { SURFACE }}{\left(\mathrm{mm}^{2}\right)}$ & $\frac{\text { SURFACE }}{\left(\mathrm{mm}^{2}\right)}$ \\
\hline 1) Normal controls & 16 & $758 \pm 1604$ & $331 \pm 523$ \\
\hline 2) Neurological controls & 6 & $690 \pm 560$ & $857 \pm 867$ \\
\hline 3) Friedreich's ataxia (Ia) & 17 & $4389 \pm 4306$ & $4739 \pm 5386$ \\
\hline 4) Roussy-Levy (IIa) & 6 & $2357 \pm 1309$ & $3167 \pm 990$ \\
\hline $\begin{array}{l}\text { 5) Spinocerebellar degene- } \\
\text { rations (dominant) }\end{array}$ & 5 & $698 \pm 625$ & $1303 \pm 1920$ \\
\hline
\end{tabular}

TABLE 2

GLUCOSE-PYRUVATE TEST

IN RELATIVES OF FRIEDREICH'S ATAXIA (mean \pm S.D.)

\begin{tabular}{|c|c|c|c|c|}
\hline \multicolumn{2}{|c|}{ GROUP } & \multirow{2}{*}{$\begin{array}{l}\underline{N} \\
16\end{array}$} & \multirow{2}{*}{$\begin{array}{c}\frac{\text { ABNORMAL PYRUVATE }}{\frac{\text { SURFACE }}{\left(\mathrm{mm}^{2}\right)}} \\
758 \pm 1604\end{array}$} & $\frac{\text { ABNORMAL LACTAT }}{\frac{\text { SURFACE }}{\left(\mathrm{mm}^{2}\right)}}$ \\
\hline 1) & Normal controls & & & $331 \pm 523$ \\
\hline 2) & $\begin{array}{l}\text { Friedreich's ataxia } \\
\text { (Group Ia) }\end{array}$ & 17 & $4389 \pm 4306$ & $4739 \pm 5386$ \\
\hline 3) & Relatives of Group Ia patients & 14 & $1834 \pm 2320$ & $1885 \pm 2432$ \\
\hline & a) Asymptomatic siblings & 4 & $616 \pm 770$ & $1747 \pm 2161$ \\
\hline & b) Parents & 10 & $2321 \pm 2015$ & $1940 \pm 1789$ \\
\hline & c) Fathers & 5 & $2878 \pm 3176$ & $1119 \pm 1298$ \\
\hline & d) Mothers & 5 & $1765 \pm 2028$ & $2761 \pm 3510$ \\
\hline
\end{tabular}

4) Sub-groups of Group Ia

A) High_pyruvate $(3$ kindreds)
a) Patients
$10021 \pm 3205$
$9629 \pm 7923$
b) Parents
$3832 \pm 2264$
$3181 \pm 2815$
B) Low_pyruyate (2 kindreds)
a) Patients
$1986 \pm 1804$
$56 \pm 111$
$3472 \pm 3192$
$78 \pm 111$ 
where it is pathological (see previous paper).

b) Determination of thiaminepyrophosphate, through the transketolase method, was carried out in 11 normal age-matched control subjects and 11 patients from Group Ia (typical Friedreich's ataxia). The results are given in Table 4. A control patient was al- ways studied on the same day, and at the same time as an ataxic patient. Each determination was carried out in duplicate. It is seen that the two groups do not differ, with or without the addition of TPP. There is no evidence of thiamine deficiency in the patients studied.

c) Pyruvate oxidation in white blood cells. The production of ${ }^{14} \mathrm{CO}_{2}$ from

\section{TABLE 3 \\ CHARACTERISTICS OF HIGH \\ AND LOW PYRUVATE KINDREOS \\ (mean \pm S.D.)}

HIGH PYRUVATE RESPONDERS
1) Number of kindreds
2) Number of patients
3) Abnormal pyruvate surface
4) Abnormal lactate surface
5) Fasting glucose (mg\%)
6) $2 \mathrm{hr}$. P.C. glucose (mg\%)
7) fasting insulin
(normal values $23 \pm 8$ )
8) Danowski score
9) Total bilirubin (mg\%)
10) S.G.P.T. (I.U.)
11) L.D.H. (I.U.)
12) Cholesterol $(\mathrm{mg} / 100 \mathrm{ml})$
13) Triglycerides (mg/100 $\mathrm{ml}$ )
14) Verbal 1.Q.
15) Fasting pyruvate ( $\mu M / 1)$
16) Fasting lactate ( $\mu M / 1$ )

\begin{tabular}{|c|c|c|}
\hline & 3 & 2 \\
\hline & 4 & 4 \\
\hline 10,021 & \pm 3205 & $1986 \pm 1804$ \\
\hline 9,629 & \pm 7923 & $3472 \pm 3192$ \\
\hline 89.0 & \pm 3.16 & $89.0 \pm 15.9$ \\
\hline 117.0 & \pm 55.15 & $111.5 \pm 37.4$ \\
\hline 10.75 & $\pm \quad 5.7$ & $18.25 \pm 8.65$ \\
\hline 484 & $\pm \quad 310.4$ & $388 \pm 51.9$ \\
\hline 0.97 & \pm 0.22 & $1.72 \pm 0.79$ \\
\hline 12.0 & \pm 10.7 & $14.0 \pm 4.96$ \\
\hline 157 & $\pm \quad 56.3$ & $144 \pm 14.8$ \\
\hline 148 & $\pm \quad 21.1$ & $129.5 \pm 26.9$ \\
\hline 56.7 & $\pm \quad 28.9$ & $67.8 \pm 16.02$ \\
\hline 82.0 & 5.7 & $113.7 \pm 4.04$ \\
\hline 100.2 & $\pm \quad 40.5$ & $73.2 \pm 22.99$ \\
\hline 2477.5 & 1825.0 & $1755.0 \pm 266.0$ \\
\hline
\end{tabular}

TABLE 4

IRANSKETOLASE DETERMINATIONS

(mean \pm S.D.

CONTROL SUBJECTS

$(N=11)$

a) Without excess TPP

$110.4 \pm 17.6 \mathrm{mg} \%$

$113.1 \pm 17.4 \mathrm{mg} \%$
FRIEDREICH'S ATAXIA

$(N=11)$

$102.5 \pm 12.2$

$106.8 \pm 15.9$
$1-{ }^{14} \mathrm{C}$-pyruvate was measured in white blood cells of 20 fasting patients with Friedreich's ataxia (from Group Ia) and 20 age and sexmatched controls. A patient was always measured with his control. There were no significant differences observed, as recorded in Table 5. The Friedreich's cases had $123 \%$ of control activity in pyruvate oxidation within their leukocytes.

d) Oxidation of ${ }^{14} \mathrm{C}-(1)-$ pyruvate by intact fibroblasts (first laboratory). The oxidation of $\mathrm{C}$ 1 -pyruvate was measured in cultured fibroblasts from 8 typical Friedreich's ataxia cases and 8 control cases in one laboratory. The results are given in Table 6 . There is no significant difference between the two groups. Because there can be important technical variations from day to day in this type of determination, it is important to measure a patient and his own control on the same day. Otherwise 3 patients with low values $(3.5,8.7,9.6)$ would have been considered abnormal. However, that same day, the 3 matched controls read 8.7, 10.6, 12.0

e) Oxidation of $2-{ }^{14} \mathrm{C}$-pyruvate by intact fibroblasts (first laboratory). The fibroblasts from the same 8 patients and 8 controls served for the measurement of the oxidation of $2-{ }^{14} \mathrm{C}$-pyruvate in the same laboratory. The two groups of patients do not differ, Table 6 . We found 2 low values $(0.04$ and 0.06$)$, but the matched controls were also low (both 0.03). These low determinations were, however, not in the same fibreblasts as for $1-{ }^{14} \mathrm{C}-$ oxidation.

f) Oxidation of $2-{ }^{14} \mathrm{C}$ - pyruvate and uniformly labelled pyruvate in fibroblasts (second laboratory). Because of the importance of confirming or contradicting published findings on pyruvate oxidation in Friedreich's ataxia, we carried out oxidation measurements in a second laboratory from the same fibroblast cultures using slightly different methodological approaches (Melançon et al., unpublished). The oxidation of uniformly labelled ${ }^{14} \mathrm{C}$ or of $2-{ }^{14} \mathrm{C}-$ pyruvate, as well as of uniformly labelled ${ }^{14} \mathrm{C}$ or b) With excess TPP 
$1{ }^{14} \mathrm{C}$-acetate (New England Nuclear Corporation) was normal in typical and atypical Friedreich's ataxia subjects from our survey when compared to the results in probable or obligatory carriers (parents) or normal control subjects (Table 7).

g) Determination of lipoamide dehydrogenase $(L A D)$ activity

The third component $\left(\mathrm{E}_{3}\right)$ of PDH is lipoamide dehydrogenase. This was evaluated in the serum of 10 cases of Friedreich's ataxia( all from Group la) and 10 age-matched controls. A control patient was evaluated on the same day as a case of Friedreich's ataxia. As seen in Table 8, there were no differences between the two groups, when taken as a whole. However, when the patients are divided according to their pyruvate response to glucose (see above), some differences or trends can be seen. The low pyruvate responders (members of 2 kindreds) show no differences from their controls, whereas the high pyruvate responders (members of 3 kindreds), have a $29 \%$ decrease in serum LAD concentrations.
TABLE 5

PYRUVATE OXIDATION IN WHITE BLDOD CELLS

(moles ${ }^{14} \mathrm{CO}_{2} / 10,000 \mathrm{WBC} / \mathrm{hr}$ )
CONTROLS

Number of patients

Age (mean)

POH Activity

Range

20

24.8
FRIEDREICH

22.1

$(1.85 \pm 0.20) \times 10^{-12}(2.27 \pm 0.25) \times 10^{-12}$
$(0.50-3.81) \times 10^{-12}(0.78-4.63) \times 10^{-12}$

TABLE 6

OXIDATION OF PYRUVATE BY FIBROBLASTS

(CPM/mg protein/min)

\begin{tabular}{|c|c|c|}
\hline & $\frac{\text { CONTROLS }}{(N=8)}$ & $\frac{\text { FRIEDREICH }}{(N=8)}$ \\
\hline $\begin{array}{c}\text { Oxidation of }{ }^{14} \mathrm{C}-(1)- \\
\text { Pyruvate. range }\end{array}$ & $\begin{array}{c}15.72 \pm \\
(8.7-24.5)\end{array}$ & $\begin{array}{c}16.37 \pm \\
\left(8.7^{ \pm}-24.3\right)\end{array}$ \\
\hline $\begin{array}{c}\text { Oxidation of }{ }^{14} \mathrm{C}-(2)- \\
\text { Pyruvate range }\end{array}$ & $\begin{array}{c}0.22 \pm \\
(0.03-0.71)\end{array}$ & $\begin{array}{c}0.30 \pm \\
(0.04-1.01)\end{array}$ \\
\hline
\end{tabular}

\section{TABLE 7}

PYRUVATE OXIDATION IN FIBROBLASTS

OF FRIEDREICH'S ATAXIA (LABORATORY \# 2)

(mean + range)

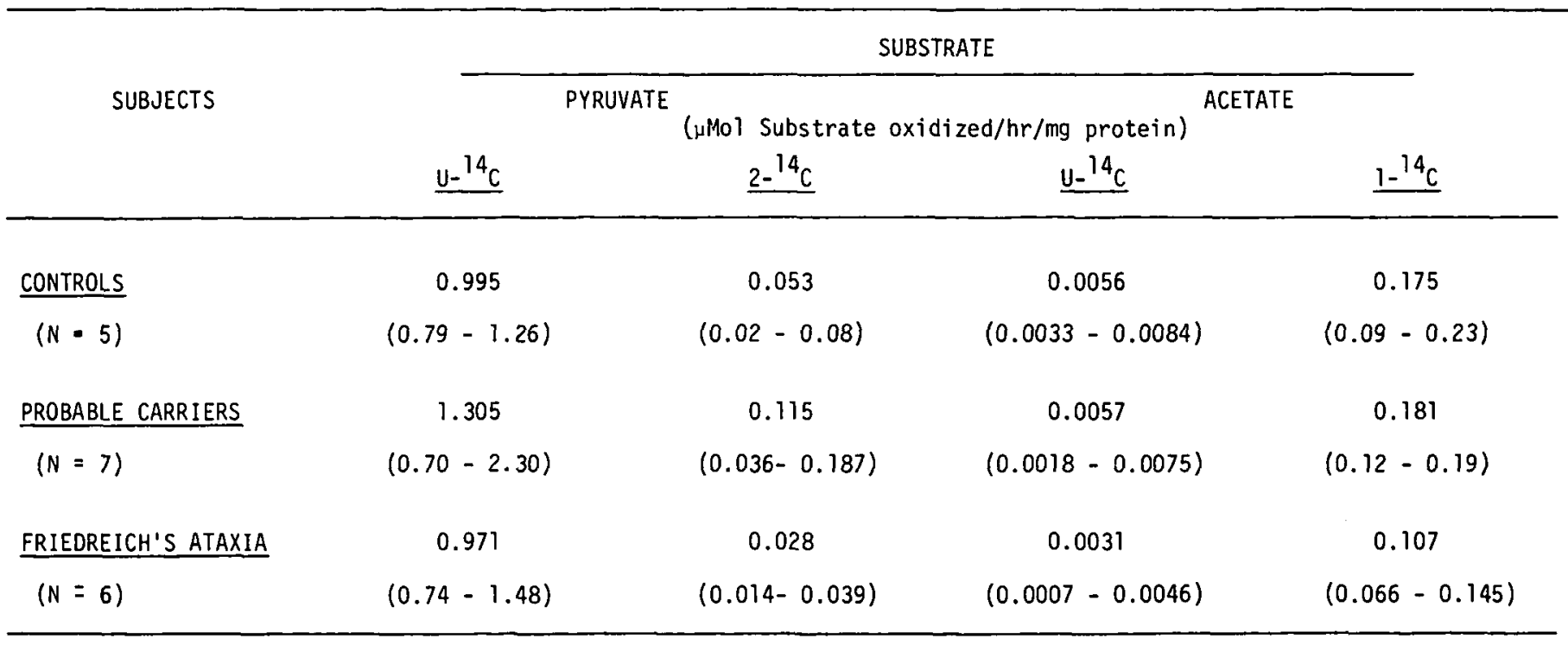




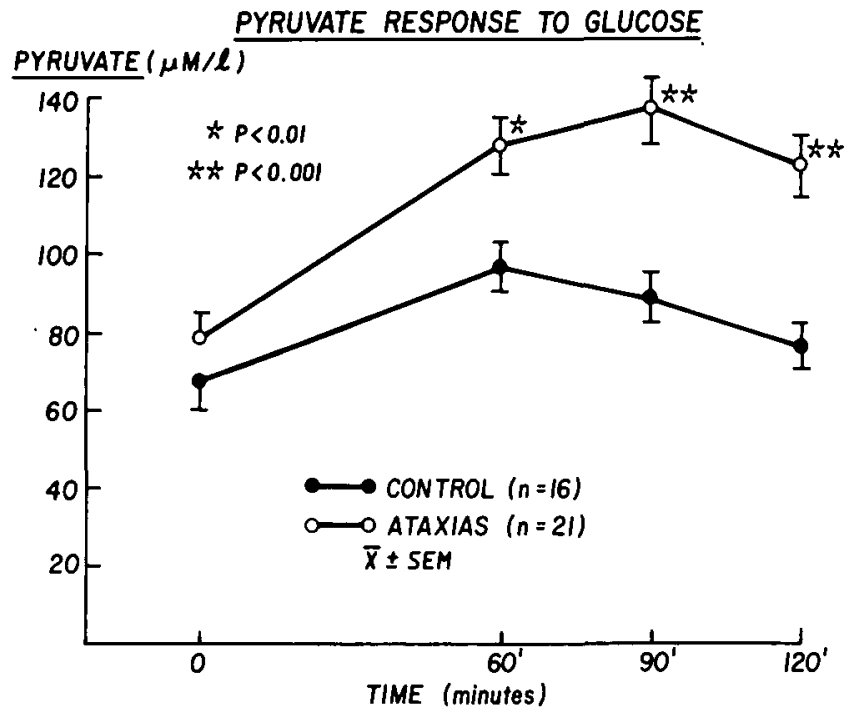

Figure 3 - Blood pyruvate response to an oral glucose load in Friedreich's ataxia and normal control subjects.

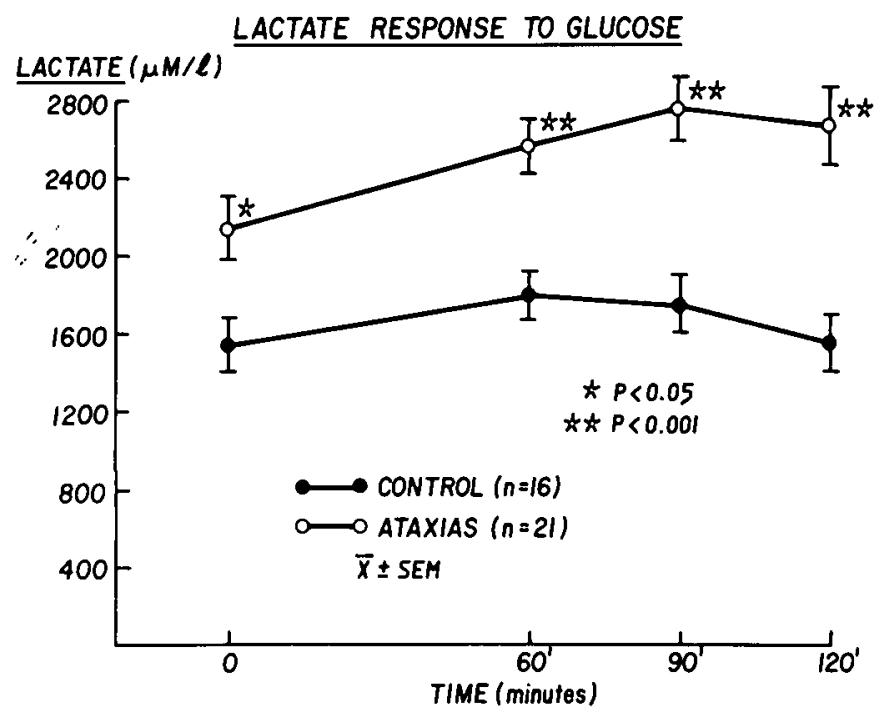

Figure 4 - Blood lactate response to an oral glucose load in Friedreich's ataxia and normal control subjects.

\section{h) Other enzymes and metabolic products}

We also investigated the SGPT, and $\mathrm{LDH}$, as well as the fasting pyruvate and lactate in both high (4 patients) and low (4 patients responders. These data are shown in Table 3 . While the enzymes do not appear to indicate differences, the fasting pyruvate and lactate levels appear to be higher in the high responders, again possibly indicating the presence of two biochemical sub-groups.

\section{i) Relationship between pyruvate results and insulin}

Figure 6 indicates the obvious and significant inverse relationship between fasting insulin levels in 14 Friedreich's ataxia patients from Group la and the abnormal pyruvate surface (in $\mathrm{mm}^{2}$ ) as calculated from the response of pyruvate to a glucose load. Higher fasting insulin levels, at rest (i.e. normal levels which are $23 \pm 8 \mu \mathrm{U} / \mathrm{ml}$ ) are associated with fewer abnormalities in pyruvate metabolism. When the insulin levels are low, pyruvate does not appear to be metabolized as fast.

It should also be noted that the 4 low responders (Table 3 ) had 1 normal, 3 type 1 insulin curves with 1 normal and 3 diabetic GTT curves,

\section{TABLE 8}

LIPOAMIDE DEHYDROGENASE ACTIVITY

\begin{tabular}{lccccccc}
\hline & \multicolumn{2}{c}{ CONTROLS } & & \multicolumn{2}{c}{ FRIEDREICH'S ATAXIA } & \\
& $N$ & Results & & $N$ & Results & Significance \\
\hline $\begin{array}{l}\text { SERUM L.A.D. } \\
\begin{array}{l}\text { a) High pyruvate } \\
\text { Responders }\end{array}\end{array}$ & 10 & $150.5 \pm 66.85$ & 10 & $125.5 \pm 43.4$ & N.S. \\
$\begin{array}{l}\text { b) Low Pyruvate } \\
\text { Responders }\end{array}$ & 5 & $162.3 \pm 70.25$ & 5 & $115.3 \pm 38.2$ & $-29 \%$ \\
& 5 & $138.8 \pm 55.71$ & 5 & $135.8 \pm 49.43$ & N.S.
\end{tabular}

while the 4 high responders all had type 3 insulin and delayed diabetic curves.

\section{j) Relationship between pyruvate} results and total bilirubin

Figure 7 illustrates the relationship between the calculated abnormal pyruvate surface and total

\section{DISTRIBUTION OF PYRUVATE} RESPONSE IN 17 "TYPICAL" FRIEORIECH'S ATAXIA (GROUP IO) CASES

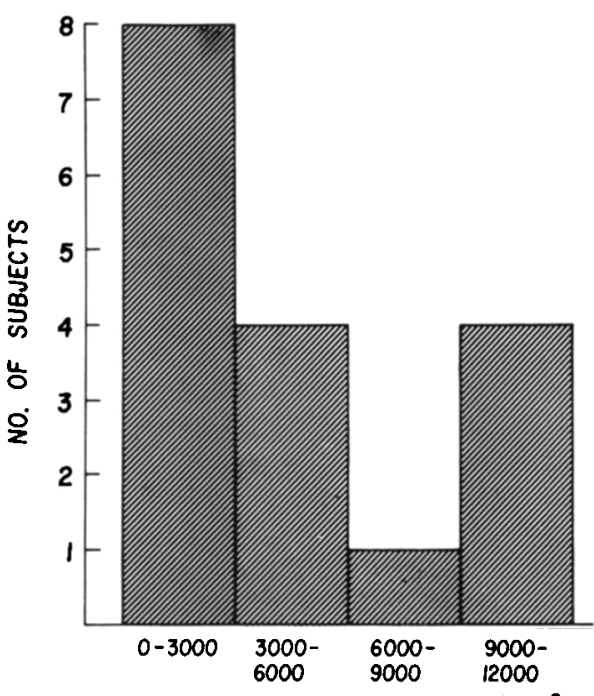

ABNORMAL PYRUVATE SURFACE $\left(\mathrm{mm}^{2}\right)$

Figure 5 - Distribution of "abnormal" pyruvate surface in Friedreich's ataxia patients after an oral glucose load. 
bilirubin values (mean $1.71 \mathrm{mg} \%$ ) in the 4 low responders and in the 4 high responders (mean $0.95 \mathrm{mg} \%$ ).

k) Relationship between normal pyruvate metabolism and age or duration of disease. The relationship between the abnormal pyruvate surface as calculated after glucose ingestion and either age or duration of the disease was evaluated in 14 Group Ia patients. We could not demonstrate any increase or decrease in the severity of the abnormality with advancing years. In one patient, still within the first year of evolution (8 year old girl), we observed one of the highest values. This would be one argument in favor of the thesis that the pyruvate oxidation defect is not secondary to the disease process.

\section{DISCUSSION}

As noted by Joiner et al. (1950), Collis and Engel (1968), Shahani et al. (1970) and more recently by Blass and collaborators (1976), familial ataxia can occur in a variety of inborn errors of metabolism. Thus, disorders of lipid metabolism such as Refsum's disease with phytanic acid storage (Steinberg, 1972), A$\beta$-lipoproteinemia (Fredrickson et al., 1972) varieties of moderate de- ficiencies in $\beta$-galactosidase (Wenger et al., 1974) or juvenile arylsulfatase A deficiencies (Pilz, 1969) can manifest themselves with ataxia. The same can be said for a number of specific aminoacidurias such as Hartnup disease (Jepson, 1972), intermittent maple-syrup-urine disease (Dancis and Levitz, 1972), or $\gamma$-glutamyl-cysteinyl transferase deficiencies (Meister, 1974). To date, it has not been possible to isolate a common denominator to these genetically and phenotypically different entities. In many of these disorders, the clinical syndrome of spinocerebellar degeneration is observed in a few patients.

For many years, it has been recognized that pyruvate oxidation might be impaired in diseases showing evidence of ataxia and peripheral neuropathy. This has been known since 1950 when abnormal pyruvate responses to glucose were reported by Joiner and collaborators (1950) in polyneuropathies, motor neuron disease and a few myopathies. The non-specificity of this response was illustrated by the fact that it could also be observed in simple obesity (Doar, and Wynn, 1970). Pyruvate oxidation, as seen in Figures 1 and 2, involves a complex series of enzymatic reactions. It is not surpris- ing that specific defects in one or more steps of this cascade have been reported in man. The first step (E1-pyruvate decarboxylase) can be impaired in thiamine deficiency (Dreyfus and Victor, 1961), and elemental or organic mercury poisoning (Thompson and Whittaker, 1947; Menon and Kark, 1976), all diseases with prominent ataxia. Specific apoenzyme deficiencies in $E_{1}$ or $E_{2}$ have also been noted as genetic entities (Blass et al., 1970, 1971, 1972, 1976), but they are usually accompanied by congenital or acquired lactic acidosis, high fasting pyruvate and lactate levels and most patients do not survive infancy (Londsdale, 1969; Farrell et al., 1975; Strömme et al., 1976). In some cases, in addition to the lactic acidosis, one can find elevated levels in blood and urine of alanine, the transamination product of pyruvate. This was demonstrated by Dunn and Dolman (1969) in a child with the clinical signs of Friedreich's ataxia and the usual changes of subacute necrotizing encephalomyelopathy.

In a recent series of papers, Blass and co-workers (Kark et al., 1974; Blass et al., 1976; Reynolds and Blass, 1976) have studied in detail a specific and genetically determined defect in pyruvate oxidation affect-
RELATIONSHIP PYRUVATE METABOLISM - INSULIN IN FRIEDREICH'S ATAXIA

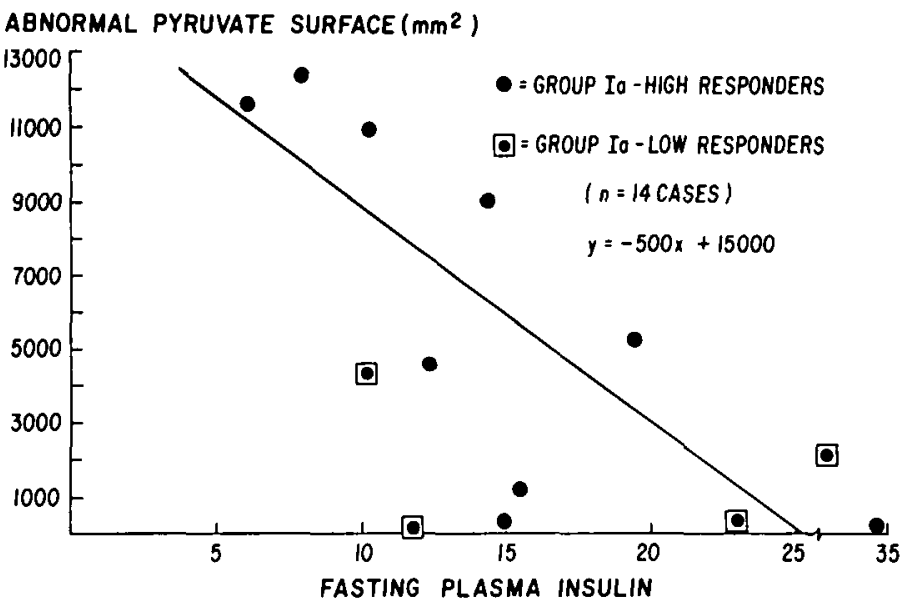

Figure 6 - Relationship between fasting insulin responses and the abnormal pyruvate response (in $\mathrm{mm}^{2}$ ) after an oral glucose load in Friedreich's ataxia.
RELATIONSHIP PYRUVATE - TOTAL BILIRUBIN

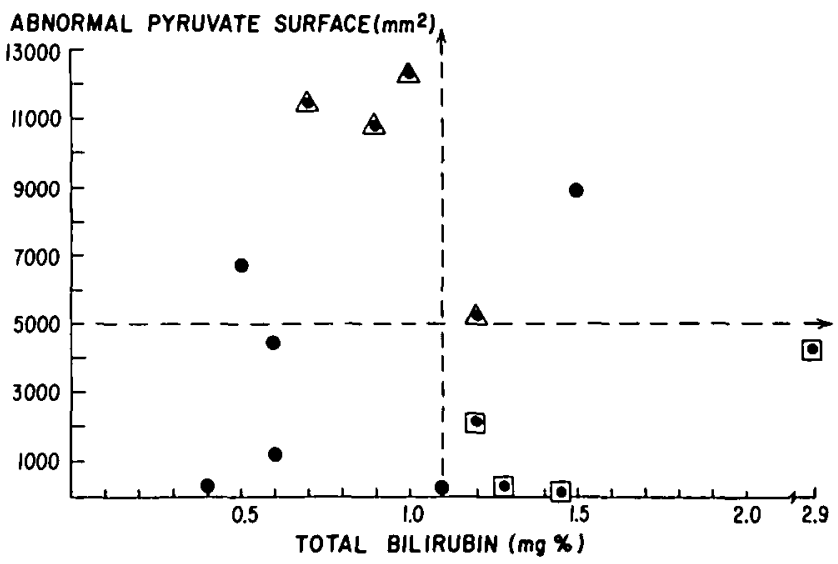

Figure 7 - Relationship between the abnormal pyruvate response (in $\mathrm{mm}^{2}$ ) after an oral glucose load and blood total bilirubin values in Friedreich's ataxia. Triangle containing dot $=$ high responders, square containing dot $=$ low responders, dot = Friedreich's Ataxia, Group Ia. 
ing particularly the $E_{3}$ (lipoamide dehydrogenase) apoenzyme component of the pyruvate dehydrogenase (PDH) complex, in Friedreich's ataxia. However, closer examination of their papers suggests that should such a defect exist (it is unlikely because one would imagine more severe damage to the brain oxidative pathways and therefore to the function of all cells), it cannot be specific for all Friedreich's ataxia cases. In their first paper (Kark et al., 1974), the authors studied 57 subjects, of which 8 were controls, 11 myopathies, 19 lower motor neuron disorders and 19 ataxias. Of the latter, only 7 had the classical features of Friedreich's ataxia. Nevertheless, when pyruvate oxidation in muscle was studied (o.5 $\mathrm{M} / \mathrm{gm}$ non collagenous protein/hr. being the lowest limit of normal), low values were found in only 4 of the 7 Friedreich's, in 4 of the 12 other ataxias and in 8 of the 19 neuropathies. There was no correlation between the level of pyruvate oxidation and the severity of neuropathic changes in muscle. It is important to note that $3 / 7$ Friedreich's were normal. No emphasis on this fact was made by Kark et al. (1974). From the same paper, it is seen that blood pyruvate response to a glucose load was abnormal in only 2 of the 3 Friedreich's with low muscle pyruvate oxidation rates. In a later paper (Blass et al., 1976), the same authors attempted to define the biochemical abnormality more precisely by studying, in disrupted cultured fibroblasts of 5 patients, the total pyruvate dehydrogenase complex, the $E_{1}$ component and, as a comparison, the 2-oxoglutarate ( $\alpha \cdot$ keto-glutarate) complex. The results obtained in these highly selected patients show a $43 \pm 4 \%$ reduction in total PDH activity as well as a $50 \pm 2 \%$ reduction in the 2-oxo-glutarate dehydrogenase complex with normal cytochromeC-oxidase activity. On the other hand, the $E_{1}$ (pyruvate decarboxylase) component when tested specifically, was found within normal limits. Mixing experiments gave no evidence of soluble enzyme inhibitors or activators, and the addi- tion of excess substrate or co-factor did not ameliorate the deficiencies. Blass et al. (1976) observed that the only common denominator between PDH and 2-oxo-glutarate dehydrogenase was the $E_{3}-$ component: lipoamide dehydrogenase, the part of the complex involved in regeneration of the activity. They concluded that the disturbance probably results from a genetically determined defect since it persisted in tissue culture, and that this mutation involves the apoenzyme protein moiety of lipoamide dehydrogenase, in some patients with Friedreich's ataxia.

Such a conclusion would be an important finding if it could be confirmed in either a clearly delimited sub-group of patients or generally in Friedreich's ataxia. Unfortunately, in our study we are unable to be as specific. Firstly, in vivo, we could demonstrate abnormalities in glucose metabolism in approximately $40 \%$ of our typical Friedreich's ataxia patients. Not all patients responded to a glucose load with an abnormal pyruvate oxidation rate. We could clearly delineate two subgroups: "high responders" and "normal or low responders". This response appeared to be genetically determined, for the parents of the high responders had high responses, while the parents of the normal responders had low responses. Furthermore, the high responders had a greater incidence of abnormal GTT, of clinical diabetes, of high fasting pyruvate and lactate levels and finally of high urinary alanine than the low or normal responders. This was less clearly reflected in a lower mean I.Q. of the high responders. In vivo studies confirm that there exists a sub-group of typical Friedreich's ataxia patients who have impaired oxidation of pyruvate. It should, however, be noted that this is not the case for all typical Friedreich's ataxia cases studied. It is possible to show the complete phenotype of Friedreich's ataxia without evidence of low pyruvate oxidation as measured in vivo. The specificity and primary importance of this defect in the production of Friedreich's ataxia is further limited by the observation that a similar defect in pyruvate oxidation can be found (equally in vivo) in other ataxias and chronic neuropathies.

Our in vitro studies differ from those of Blass and collaborators (1976) and indicate that the findings of these authors can, at best, be specific to a very small sub-group of ataxic patients and, with the other interpretation, be subject to complete reevaluation as regards their application to typical Friedreich's ataxia. We were unable to demonstrate any major in vitro impairment of either the whole PDH complex or of its $E_{1}$, and $E_{3}$ components. $E_{1}$ activity was found normal (in agreement with Blass) from $1-{ }^{14} \mathrm{C}$-pyruvate oxidation in both leukocytes and cultured fibroblasts. Thiamine pyrophosphate (TPP) as measured with the transketolase method was also normal. Oxidation of either $2-{ }^{14} \mathrm{C}-$ pyruvate or uniformly-labelled ${ }^{14} \mathrm{C}$-pyruvate was measured on cultured fibroblasts, by two slightly different methods in two different laboratories, and was found to be normal, indicating that the total complex, and by subtraction the $E_{2}$ component, was normal in our typical patients. Since our control values for such measurements are the same as those of Kark et al. (1974), we conclude that the difference probably lies in the selection of patients for study and not in methodology. Finally, our own studies of the $E_{3}$ component (lipoamide dehydrogenase, $\mathrm{LAD}$ ) in serum indicate a $29 \%$ deficiency only in the high pyruvate responder sub-group of typical Friedreich's ataxia patients (Table 7). On the other hand, the normal or low pyruvate responders show no difference from controls. We must conclude that a LAD defect of this magnitude, if it exists, is not specific to all cases of Friedreich's ataxia, and is unlikely to be due to a genetic mutation in the LAD apoenzyme, as proposed by Blass et al. (1976).

\section{Our first conclusions are:}

1) that pyruvate oxidation, in vivo, is low in a definite sub-group of typical Friedreich's ataxia patients but not in all such patients; 
2) that in vitro studies in most cases of Friedreich's ataxia from the above two sub-groups are unable to demonstrate a major defect in the $\mathrm{E}_{1}$, $E_{2}$, or $E_{3}$ components of the PDH complex, except for a partial deficiency in LAD in some, but not all patients. Perhaps the patients studied by Blass et al. (1976) belong to such a sub-group.

3 ) that the magnitude and reversability of the pyruvate oxidation deficiency (within a few hours after a glucose load and with normal resting values), when it exists, is likely due to a defect in PDH regulation rather than to a genetic mutation in the apoenzyme components of the complex. This regulatory defect could be genetically determined.

What could determine this regulatory defect in some cases of Friedreich's ataxia? Reed (1974) and Wieland (1972) have studied in detail the regulation of the pyruvate dehydrogenase complex and have shown that the enzyme complex exists (Figure 2) in an active and an inactive (phosphorylated) state. Transformation from one form to the next is dependent upon two enzymes (a magnesium and ATP-dependent kinase for inactivation and a calcium-magnesium dependent phosphorylase for activation) and a number of co-factors (magnesium, ATP/ADP ratio, calcium). Furthermore, this transformation is metabolically controlled by end product (acetyl co-enzyme A and NAD) inhibition. In turn, the concentration of acetyl co-enzyme A depends on the free fatty acid production regulated by insulin. There is no question that in vivo pyruvate oxidation can be impaired by one or more of the above factors. Since diabetes is frequent in Friedreich's ataxia, it is not impossible that total PDH activity in vivo will be inversely proportional to the availability of insulin. Such was the case as demonstrated in Figure 6 . The patients with clinical diabetes were generally those who had more evidence of defective pyruvate oxidation. However, abnormal glucose tolerance curves, with normal insulin responses, were also seen in low pyruvate responders.

It is possible that the PDH defect revealed in vivo under the above mentioned metabolic stress situations (glucose load), is worse in patients with impaired glucose or insulin metabolism, but they mask an underlying genetic partial deficiency in the functioning of the complex. We have seen that the regenerative component (LAD; lipoamide dehydrogenase, $E_{3}$ ) is the only common denominator between pyruvate dehydrogenase and 2-oxo-glutarate (or $\alpha \cdot$ keto-glutarate) dehydrogenase and that it appeared to be partially impaired in our high pyruvate responders and probably markedly blocked in the patients studied by Blass et al. (1976). If the regenerating component of the complex is impaired alone without the $E_{1}$ and $E_{2}$ components, it would explain that the deficiency in pyruvate oxidation would appear only under conditions of "metabolic stress" (a glucose load). It would certainly be made worse by the added presence of the metabolic changes inherent to diabetes (low insulin, high free fatty acids, etc.).

In the case of $L A D$, we are reluctant, in the cases we studied, to postulate, as was done by Blass et al. (1976), an apoenzyme mutation of such low amplitude (less than $30 \%$ reduction in activity in the serum). On the other hand, we could implicate a regulatory defect (possibly genetically determined) which would impair LAD's own recuperation. The nature of this regulatory defect is as yet unknown, but it could implicate the binding or interaction of NAD or of FAD to the dihydrolipoyl dehydrogenase molecule in a manner similar to the calcium regulated TPP binding at the $E_{1}$ component or the calcium regulated phosphatase binding at the transacetylase $\left(E_{2}\right)$ step. Preliminary evidence does implicate calcium and/or pyridoxal phosphate in the binding of NAD to the $E_{3}$ moiety. Neither of these cofactors were tested in the experiments of Blass and co-workers (1976), and therefore a defect in their in vivo availability cannot be ruled out.

In conclusion, we confirm that some, but not all, patients with typical Friedreich's ataxia show a deficiency of in vivo pyruvate oxidation.
This deficiency is worse in a subgroup of patients with abnormalities in glucose metabolism and low fasting insulin levels. It appears globally to be genetically determined. To account for the equally low 2-oxo-glutarate dehydrogenase activity in some patients, the $E_{3}$ (Lipoamide dehydrogenase) component of both complexes could be deficient. We do not accept the hypothesis that this defect results from a genetic mutation in the apoenzyme of LAD, but propose instead that it is secondary to a regulatory dysfunction in the activity of LAD, perhaps linked to calcium and/or pyridoxal phosphate binding and perhaps genetically transmitted. It is uncertain whether all patients with Friedreich's ataxia have this LAD regulatory defect and whether a deficiency in pyruvate oxidation is more than a secondary accompaniement of the disease.

\section{ACKNOWLEDGMENTS}

The studies reported in this paper were supported in part by L'Association Canadienne de l'Ataxie de Friedreich, la Fondation Jeanne-Mance and the Medical Research Council of Canada. The authors extend their appreciation for excellent technical assistance to Mrs. F. Landreville, Miss F. Laviolette, Miss M. Charbonneau and Mr. F. Bélanger.

\section{REFERENCES}

BARBEAU, A. (1975). Preliminary studies on pyruvate metabolism in Friedreich's ataxia. Trans. of Am. Neurol. Assoc., 100, 164-165.

BLASS, J. P., AVIGAN, J., and UHLENDORF, B.S. (1970). A defect in pyruvate decarboxylase in a child with an intermittent movement disorder. J. Clin. Invest., 49, 423-432.

BLASS, J. P., AVIGAN, J., and UHLENDORF, B: S. (1970). A defect in pyruvate patient with pyruvate decarboxylase deficiency Arch. Neurol, 25, 449-460.

BLASS, J. P., SCHULMAN, J. D., and YOUNG, D. S. (1972). An inherited defect affecting the tricarboxylic acid cycle in a patient with congenital lactic acidosis. J. Clin. Invest., 51, 1845-1851.

BLASS, J. P., KARK, R. A. P., and MENON, N. K. (1976). Low activities of the pyruvate and oxoglutarate dehyd. rogenase complexes in five patients with Friedreich's ataxia. New Engl. J. Med., 295, 62-67.

COLLIS, W. J., and ENGEL, W. K. (1968). 
Glucose metabolism in five neuromuscular disorders. Neurology, 18, 915-925.

DANCIS, J., and LEVITZ, M. (1972). Abnormalities of branched-chain amino acid metabolism. In: The Metabolic Basis of Inherited Disease, 3rd Edition (Stanbury, J. B., Wyngaarden, J. B. and Fredrickson, D. S., eds.). McGraw-Hill, New York, pp. 426-439.

DREYFUS, P. M., and VICTOR, M. (1961). Effects of thiamine deficiency on the central nervous system. Am. J. Clin. Nutr., 9, 414-425.

DUNN, H. G., and DOLMAN, C. L. (1969). Necrotizing encephalomyelopathy. Report of a case with relapsing polyneuropathy and hyperalaninemia and with manifestations ressembling Friedreich's ataxia. Neurology (Minneap.), 19, 536-550.

FARRELL, D. F., CLARK, A. F., and SCOTT, C. R. (1975). Absence of pyruvate decarboxylase in man: a cause of congenital lactic acidosis. Science, 187, 1082-1084.

FREDRICKSON, D. S., GOTTO, A. M., and LEVY, R. I. (1972). Familial Lipoprotein deficiency ( $\alpha \beta$-lipoproteinemia, hypo-lipoproteinemia, and Tangier disease. In: The Metabolic Basis of Inherited Disease, 3rd edition, (Stanbury, J. B., Wyngaarden, J. B., and Fredrickson, D. S., eds). McGraw-Hill, New York, pp. 498-530.

HEWER, R. L., and ROBINSON, N. (1968). Diabetes mellitus in Friedreich's ataxia. $\mathbf{J}$. Neurol. Neurosurg. Psychiat., 31, 226-231.

JEPSON, J. B. (1972). Hartnup disease. In: The Metabolic Basis of Inherited Disease, 3rd edition, (Stanbury, J. B., Wyngaarden, J. B. and Fredrickson, D. S., eds). McGraw Hill, New York, pp. 1486-1503.

JOHNSON, J. L. (1975). Compartmentation of $\left(\mathrm{U}-{ }^{14} \mathrm{C}\right)$ proline metabolism in the dorsal root ganglion. Contrasts with the ventral spinal cord gray and cerebral cortex. Brain Res., 96, 192-196.
JOHNSON, J. L. (1976). A comparative analysis of compartmentation of metabolism in the dorsal root ganglion and ventral spinal cord gray using $\left(\mathrm{U}-{ }^{14} \mathrm{C}\right.$ ) glucose, $\left(2-{ }^{14} \mathrm{C}-\right)$ glucose, $\left(6-{ }^{14} \mathrm{C}\right)$ glucose, $\left(3,4-{ }^{14} \mathrm{C}\right)$ glucose, $\mathrm{NaH}^{14} \mathrm{CO}$, and $\left(2-{ }^{14} \mathrm{C}\right)$ pyruvate. Brain Res., 101, 523-532.

JOINER, C. L., McARDLE, B., and THOMPSON, R. H. S. (1950). Blood pyruvate estimations in diagnosis and treatment of polyneuritis. Brain, 73, 431-452.

KARK, R. A. P., BLASS, J. P., and ENGEL, W. K. (1974). Pyruvate oxidation in neuromuscular diseases - Evidence of a genetic defect in two families with the clinical syndrome of Friedreich's ataxia. Neurology, 24, 964-971.

LONDSDALE, D., FAULKNER, W. R., PRICE, J. W., and SMEBY, R. R. (1969). Intermittent cerebellar ataxia associated with hyperpyruvic acidemia, hyperalaninemia and hyperalaninuria. Pediatrics, 43, 1025-1034.

MEISTER, A. (1974). The $\gamma$-glutamyl cycle: diseases associated with specific enzyme deficiencies. Ann. Intern. Med., 81, 247-253.

MENON, N. K., and KARK, R. A. P. (1976). Inhibition of oxidation in chronic alkylmercury poisoning. Proc. Ann. Soc. Neurochem., 7, 151.

PELLEY, J. W., GWYNNE, H. L., TRACY, C. L., and FRANK, F. H. (1976). Lipoamide dehydrogenase in serum: a preliminary report. Clinical Chemistry, 22, 275-277.

PILZ, H. (1969). Clinical morphological and biochemical aspects of sphingolipidoses. Neuropaediatric, 1, 383-427.

PODOLSKY, S., and SHEREMATA, W. A. (1970). Insulin-dependent diabetes mellitus and Friedreich's ataxia in siblings. Metabolism, 19 555.561.
REED, L. J., and COX, D. J. (1966). Macromolecular organization of enzyme systems. Ann. Rev. Biochem., 35, 57-84.

REED, L. J. (1974). Multienzyme complexes. Accounts of Chemical Research, 7, 40-46.

REYNOLDS, S. F., and BLASS, J. P. (1976). A possible mechanism for selective cerebellar damage in partial pyruvate dehydrogenase deficiency. Neurology, 26, 625-628.

SHAHANI, B., DAVIES-JONES, G. A. B., and RUSSEL, W. R. (1971). Motor neurone disease - Further evidence for an abnormality of nerve metabolism. J. Neurol. Neurosurg. Psychiat, 34, 185-191.

STEINBERG, D. (1972). Phytanic acid storage disease (Refsum's disease). In: The Metabolic Basis of Inherited Disease, 3rd Edition, Stanbury, J. B., Wyngaarden, J. B. and Fredrickson, D. S., eds), McGraw-Hill, New York, pp. 833-853.

STRÖMME, J. H., BORUD, O., and MOE, P. J. (1976). Fatal lactic acidosis in a newborn attributable to a congenital defect of pyruvate dehydrogenase. Pediat. Res, 10 , 60-66.

THOMPSON, R. H. S., and WHITTAKER, V. P. (1947). Antidotal activity of British Anti-lewisite against compounds of antimony, gold and mercury. Biochem. J., 4I, 342-346.

THOREN, C. (1962). Diabetes mellitus in Friedreich's ataxia. Acta Paediat. (Uppsala), 51, suppl. 135, 239-247.

WENGER, D. A., GOODMAN, S. I., and MYERS, G. G. (1974). -galactosidase deficiency in young adults. Lancet, 2 , 1319-1321.

WIELAND, O., HELMREICH, E., and HOLZER, H. (Eds.). (1972). Metabolic interconversion of enzymes. Springer Verlag, Heidelberg. 